\title{
Re-condensation from an ADAF into an inner disk: the intermediate state of black hole accretion?
}

\author{
F. Meyer ${ }^{1}$, B. F. Liu ${ }^{2}$, and E. Meyer-Hofmeister ${ }^{1}$ \\ 1 Max-Planck-Institut für Astrophysik, Karl- Schwarzschildstr. 1, 85740 Garching, Germany \\ e-mail: [frm; emm] @mpa-garching.mpg.de \\ 2 National Astronomical Observatories/Yunnan Observatory, Chinese Academy of Sciences, PO Box 110, Kunming 650011, \\ PR China \\ e-mail: bfliu@ynao.ac.cn
}

Received 7 August 2006 / Accepted 13 October 2006

ABSTRACT

\begin{abstract}
Context. Accretion onto galactic and supermassive black holes occurs in different modes, which are documented in hard and soft spectral states, commonly attributed to an advection-dominated flow (ADAF) inside a truncated disk and standard disk accretion, respectively. At the times of spectral transition an intermediate state is observed, for which the accretion flow pattern is still unclear. Aims. We analyze the geometry of the accretion flow when the mass flow rate in the disk decreases (soft/hard transition) and evaporation of gas into the coronal flow leads to disk truncation.

Methods. We evaluate the physics of an advection-dominated flow affected by thermal conduction to a cool accretion disk underneath. Results. We find re-condensation of gas from the ADAF into the underlying inner disk at distances from the black hole and at rates, that depend on the properties of the hot ADAF and vary with the mass accretion rate. This sustains an inner disk for longer than a viscous decay time after the spectral transition occurred, in accordance with the spectra that indicate cool gas in the neighborhood of the accreting black hole. The model allows us to understand why Cyg X-1 does not show hysteresis in the spectral state transition luminosity that is commonly observed for X-ray transient sources.

Conclusions. Our results shed new light on the complex mass flow pattern during spectral state transition.
\end{abstract}

Key words. accretion, accretion disks - black hole physics - X-rays: binaries - stars: individual: Cyg X-1

\section{Introduction}

Accretion onto compact objects occurs in different modes. With the investigation of radiatively inefficient accretion flows (Narayan \& Yi 1994), the observed spectra of low-mass X-ray binaries and Active Galactic Nuclei (AGN) could be understood, at low luminosities, as arising from a hot advection-dominated accretion flow (ADAF). The ADAF model or its variants provide qualitative understanding of this hard spectrum from optically thin emission (Narayan 2005). At high luminosities the accreting gas forms a standard accretion disk (Shakura \& Sunyaev 1973) reaching inward to the last stable orbit, producing a soft multi-color black body spectrum.

But from the large number of observations for X-ray binaries during the last years (McClintock \& Remillard 2006) it became obvious that for many sources the spectra document a highly complex appearance of accretion processes. Intermediate states were found, especially in connection with the change between the hard and soft spectral state (in both directions), or even the very high state, in both kind of sources, neutron star and black hole transients (Psaltis 2006). Hardness-intensity diagrams, e.g. the diagram for the outburst of GX 339-4 in 2002/2003 (Belloni et al. 2005), show that the evolution of hardness with count rate is different during outburst rise and decay, indicating differences in the accretion flows via disk and ADAF. Similar changes in hardness are visible in diagrams for XTE J1650-500 (Homan et al. 2003) and $4 \mathrm{U}$ 1630-47, (Tomsick et al. 2005). The variety in diagrams for the hard/soft change can be seen from the recent compilation of Gierliński \& Newton (2006). Kalemci et al. (2003) in their work on black hole X-ray transients during outburst decay characterize the observed features as "several global patterns of evolution for spectral and temporal parameters before, during, and after the transition". In general the Fe $\mathrm{K} \alpha$ line observed from stellar black holes and AGN, the reflection component, and also the timing properties might point to emission from cool gas in the innermost region or from the edge of the disk.

Our work concerns the pattern of accretion flow at the time of spectral state transition. It is not yet clear how the accretion flow changes from one mode to the other, e.g. from disk accretion inward to the last stable orbit to a truncated disk with a hot advection-dominated flow in the inner region. We investigate this change starting from the picture that gas evaporated that from the disk feeds the coronal flow. What happens when the mass flow rate in the disk decreases (as during decline from an outburst) and becomes lower than the evaporation rate maximum? Then only a hot flow exists. We discuss that the disk reaching inward to the last stable orbit breaks up where the evaporation efficiency is maximal (at a distance of some hundred Schwarzschild radii $\left.\left(R_{\mathrm{S}}=2 G M / c^{2}\right)\right)$. Farther inside, the cool disk would still exist. however,But not being fed by a connection to the outside disk, such a relic inner disk would disappear in a viscous time. Apparently observed intermediate states last longer. An inner disk can then only survive if it is kept up by condensation of gas from the ADAF into the disk. Since these intermediate states are poorly understood at the present time we consider, as a first step, a new situation: can cool gas exist underneath an ADAF? We discuss the physics for this kind of accretion flow in an analytical model. To include more detailed physics, an evaluation by numerical simulation has to be performed. We cannot yet answer 
in detail the question of how such cool gas could be recognized in the observed spectra.

In the past the difficulty in interpreting the spectral observations of transient low-mass X-ray binaries (LMXB) led to the question of whether the observations indicate "two independent simultaneous accretion flows", as discussed by Smith et al. (2002) and Pottschmidt et al. (2006). But whatever the geometrical configuration is in which accretion would happen simultaneously via a hot flow and via a cool disk, at the same distance these flows would not be independent of each other. This especially concerns thermal conduction. An interaction with cold clumps embedded in the hot flow causing thermal conduction of the ADAF in the radial direction was discussed by Yuan \& Zdziarski (2005).

One of the key questions in the study of the hot flow above cool gas is whether heat can be drained from the upper ADAF and be radiated away, so that matter condenses onto the cool disk. We investigate the physical properties of an ADAF affected by thermal conduction to an accretion disk below the ADAF. In particular, the energy balance of the hot flow at low height above the cool disk surface where ion and electron temperature couple is important. (For a discussion of the problem see Liu et al. 2006.)

A peculiar aspect of such evaporation and condensation processes is the wide disparity between the amount of mass contained in the ADAF and in the disk, at each moment at each distance. This is a direct consequence of widely disparate temperatures in the two media. The velocities of the inward flow, proportional to the temperature, are thus also different by orders of magnitude and, for comparable mass flow rates lead to the very different column densities in ADAF and disk. How then can such a tenuous ADAF or corona, by condensation or evaporation affect and support a much more massive disk underneath? Even as a column of the ADAF loses only a fraction of its small mass during the short time that it passes over the disk surface, the underlying disk receives the contributions of many such columns before it has moved significantly inward, and the accumulated mass received can amount to a significant fraction of its own mass content.

In Sect. 2 we discuss the diversity of the outburst light curves of X-ray transients and the observations for an intermediate spectral state. In Sect. 3 we describe the accretion flow pattern related to the soft/hard spectral change. The physics of the hot flow affected by thermal conduction is treated in Sects. 4 to 6 . Using an analytical procedure we show in Sect. 7 how the energy balance in the radiating layer close to the disk surface determines whether evaporation or condensation occurs. In Sect. 8 we determine the re-condensation rate of gas from the ADAF onto an inner relic disk. The model allows us to evaluate how the condensation depends on the mass flow rate in the ADAF and the parameters involved. We draw conclusions regardingunder which circumstances condensation would lead to an inner weak disk below the ADAF, appearing in the spectrum as soft contribution. In Sect. 9 we show how our model can explain why Cyg X-1 does not have hysteresis in the transition luminosity, which is observed for all X-ray transient sources. In Sect. 10 we compare our model with observations for an intermediate state of X-ray transients, discuss the inner edge of the disk and the application to disks in AGN. Conclusions are given in Sect. 11.

A better understanding of the pattern of the accretion flow should also give insight to the observed complex timing characteristics, the occurrence and type of quasi-periodic oscillations (QPO) and the radio connections to the spectral states. Jet production corresponding to special accretion modes
(Gallo \& Fender 2005) also is part of the picture, arising from the accretion physics.

\section{The outburst light curves of X-ray transient sources}

\subsection{The cause of the mass flow changes}

McClintock \& Remillard (2006) examined 18 confirmed black hole binaries. In many sources the accretion rate seems to increase and decrease several times until finally after an outburst a quiescent phase follows. The large diversity of light curve features was documented by Chen et al. (1997), only a few sources showing the simple pattern of "fast rise-exponential decay" (FRED), but in many sources the luminosity varies in a complex way (see also the discussion by Gierliński \& Newton 2006). Two issues together create the variety of changes of luminosity and spectral states: (1) the outburst probably is caused by the ionization instability, as the dwarf nova outbursts (Meyer \& Meyer-Hofmeister 1984). The mass flow in the disk is related to the spread of heating and cooling waves initiating the changes between hot and cool disk structure. A larger disk size in X-ray transients together with irradiation leads to a more complex pattern in mass flow and outburst light curve (for the effect of a larger disk see accretion disks in symbiotic stars, Duschl 1986); (2) the mass flow rate in the disk resulting from this outburst behavior is the key parameter that determines the accretion mode, for high rates via an accretion disk everywhere (soft spectrum), for lower rates a truncated outer disk and an ADAF inside (hard spectrum).

\subsection{The intermediate spectral state}

McClintock \& Remillard (2006) redefined the X-ray states of black hole X-ray binaries in terms of quantitative criteria that utilize both X-ray energy spectra and power density spectra. They took as intermediate the spectra that did not belong to low/hard or high/soft state, nor to the very high state (sometimes a large part of the observation, e.g. 50\% throughout the 2002-2004 outburst of 4U 1630-47, Tomsick et al. 2005). We do not consider the intermediate state in the change to the very high/soft state. This latter state is of different physical nature (see the discussion by Fender et al. 2004, and the model of disk fragmentation by Meyer 2004).

The observations show that generally the intermediate state phase during the hard/soft transition (in outburst rise) is shorter than that during the soft/hard transition (in outburst decay). For the former transition durations, of 3 to 11 days were found (Corbel et al. 2004; Göğüş et al. 2004; Belloni et al. 2005).

Our work focuses on the accretion flow pattern during the latter transition. Kalemci et al. (2003) analyze the evolution of spectral and temporal properties of several galactic black hole transients during outburst decay: XTE J1650-500, GRO J1655-40, XTE J1748-288, XTE J1755-324, 4U 1630-47, XTE J1550-564, XTE J1859+226 and GX 339-4 (for this source see also Revnivtsev et al. 2000) and the spectral analysis shows a decrease of the disk component until it becomes unobservable with PCA /RXTE within 15 days after the transition. Similar times were found for GRS 1758-258 (Smith et al. 2001; Pottschmidt et al. 2006). Cyg X-1 is a special case with an intermediate state lasing for weeks (Cui et al. 1997; Zdziarski et al. 2002). The results for neutron star sources show the same trend with shorter timescales (Barret \& Olive 2002). 
coronal flow
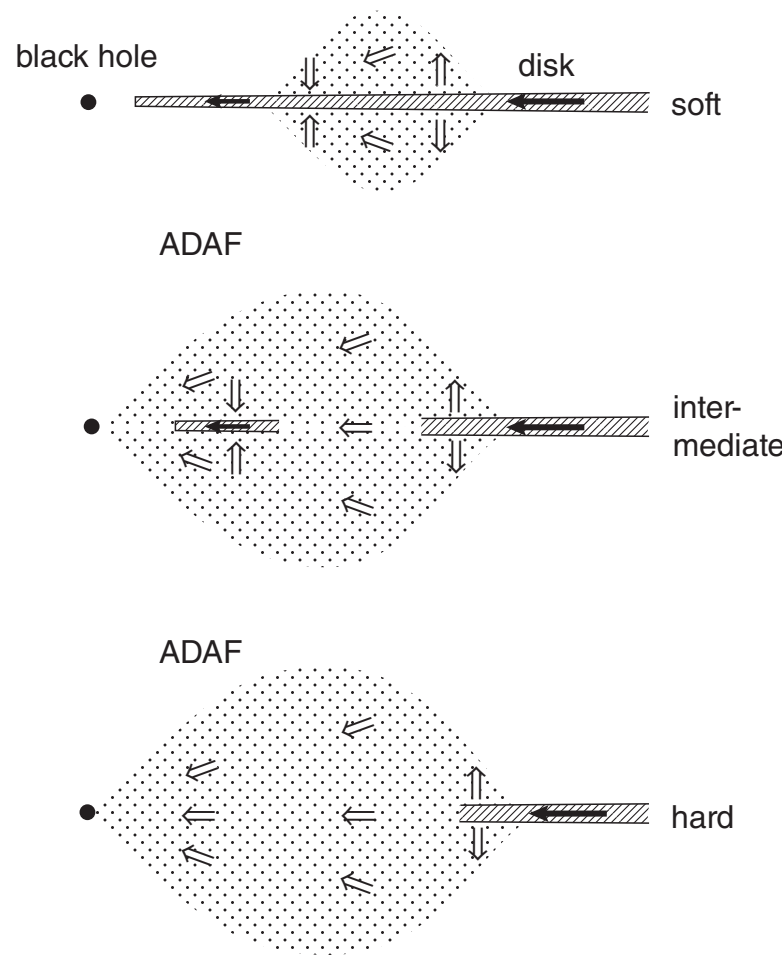

Fig. 1. Accretion flow in soft, intermediate and hard spectral state (with decreasing mass flow rate). Upper panel: in the soft state accretion occurs via the disk, some gas is evaporated to a coronal flow, but recondenses onto the disk; in the inner region only the disk flow exists, which causes the soft spectrum. Middle panel: in the intermediate state the ADAF and an inner disk contribute to the spectrum. Lower panel: in the hard state only the hot ADAF contributes to the spectrum.

\section{The accretion flow pattern at the soft/hard spectral transition: a gap in the disk?}

In earlier work on accretion onto compact objects (Meyer \& Meyer-Hofmeister 1994; Meyer et al. 2000; Liu et al. 2002; see also Różańska \& Czerny 2000a,b) we found that above the cool accretion disk a hot corona always is built up, fed by matter evaporating from the disk. The efficiency of evaporation, a siphon flow, depends on the distance from the compact star. The evaporation rate increases with decreasing distance to the center until a maximum $\left(\dot{M}_{\text {evap }}\right)_{\max }$ is reached at $R_{\text {evmax }}$. The mass flow rate in the disk determines whether the coronal flow is important. If it is higher than $\left(\dot{M}_{\text {evap }}\right)_{\max }$ the disk is essentially unaffected and reaches inward to the last stable orbit, and the hot flow is unimportant (this is the usual situation when the luminosity is high during an outburst, see also discussion in Sect. 8.3). If the mass flow rate in the disk is low, the disk is truncated where the evaporation has transfered all gas to the hot flow/ADAF (the usual situation in quiescence). According to the mass flow rate, spectral transitions occur. Note that these two transitions occur at different luminosities, an observational feature known as hysteresis in $\mathrm{X}$-ray transient light curves. This is theoretically understood as due to the different hard/soft character of the irradiation from the central region in the two cases (Meyer-Hofmeister et al. 2005; Liu et al. 2005).

When during the decrease from an outburst the mass flow rate in the disk becomes equal to the evaporation rate maximum, the disk truncation starts at the distance $R_{\text {evmax }}$, and a gap appears, filled by an ADAF, as illustrated in Fig. 1. With the further decrease of mass flow the gap should widen due to evaporation. What happens to the left-over inner cool disk? Diffusion causes a spread inwards and outwards, decreasing the amount of disk matter due to accretion onto the compact object and evaporation in outer regions. Only if matter can condense from the ADAF onto the disk can such a disk survive longer than the viscous time for these processes, which is only a few days in the inner disk. If the disks still exists the spectrum can show contributions from the ADAF and a reflection from the left-over inner cool disk.

\section{ADAF properties}

As our analysis of the hot ADAF above a left-over cool disk will show, the upper part, i.e. the main part in vertical extension, is not affected by thermal conduction to the cool disk below. We make use of the solutions of self-similar advection-dominated flows by Narayan \& Yi (1995). The properties of these flows scale with black hole mass, mass flow rate and distance from the black hole. They further depend on the viscosity and the assumed magnetic field strength. Following Narayan et al. (1998) the magnetic pressure is written

$p_{\mathrm{m}}=(1-\beta) \rho c_{\mathrm{s}}^{2}$

with $\beta$ ratio of gas pressure to total pressure, $\rho$ density and $c_{\mathrm{s}}$ isothermal sound speed. Shearing box simulations of turbulence driven by the magneto-rotational instability in a collisionless plasma by Sharma et al. (2006) yield $\beta$ values around 0.8 . We use this value in our analysis. As the ratio of specific heats of the magnetized plasma we take $\gamma=(8-3 \beta) /(6-3 \beta)$ (Esin 1997) though the true value would require a more detailed analysis.

For the chemical abundance a hydrogen mass fraction of 0.75 was used. The solutions for pressure, electron number density, viscous dissipation of energy per unit volume $q^{+}$and isothermal sound speed are (Narayan \& Yi 1995)

$$
\begin{aligned}
p & =1.87 \times 10^{16} \alpha^{-1} \mathrm{~m}^{-1} \dot{m} r^{-5 / 2} \mathrm{~g} \mathrm{~cm}^{-1} \mathrm{~s}^{-2}, \\
n_{\mathrm{e}} & =5.91 \times 10^{19} \alpha^{-1} \mathrm{~m}^{-1} \dot{m} r^{-3 / 2} \mathrm{~cm}^{-3} \\
q^{+} & =2.24 \times 10^{20} \mathrm{~m}^{-2} \dot{m} r^{-4} \mathrm{ergs} \mathrm{cm}^{-3} \mathrm{~s}^{-1}, \\
c_{\mathrm{s}}^{2} & =1.67 \times 10^{20} \mathrm{r}^{-1} \mathrm{~cm}^{2} \mathrm{~s}^{-2} .
\end{aligned}
$$

where $\alpha$ is the viscous coefficient, $m$ the black hole mass in units of solar mass $M_{\odot}, \dot{m}$ the mass flow rate in units of the Eddington accretion rate $\dot{M}_{\text {Edd }}=1.39 \times 10^{18} \mathrm{mg} / \mathrm{s}$, and $r$ the radius in units of the Schwarzschild radius $R_{\mathrm{S}}=2.95 \times 10^{5} \mathrm{~m} \mathrm{~cm}$. The ion number density is $n_{i}=n_{\mathrm{e}} / 1.077$. In an ADAF, ion and electron temperatures $T_{i}$ and $T_{\mathrm{e}}$ closely follow

$T_{i}+1.077 T_{\mathrm{e}}=1.98 \times 10^{12} \mathrm{r}^{-1} \mathrm{~K}$

and, if $T_{\mathrm{e}}$ is much smaller than $T_{i}$ this value can be taken for $T_{i}$ alone. Besides viscous heating, $q^{+}$compressive heating $q^{c}=$ $\frac{1}{(1-\beta)} q^{+}($Esin 1997) is important.

\section{The electron thermal profile}

In a pure ADAF, at radii $r<10^{3}$, the coupling between ions and electrons becomes poor. Almost all of the accretion energy stays with the ions, which reach near virial temperatures, the flow has a high vertical extent, and the density is low (see review by Narayan et al. 1998). The electrons cooled by bremsstrahlung and synchrotron(-Compton) radiation stay at temperatures around $10^{9.5} \mathrm{~K}$. Radiation losses are very weak. 


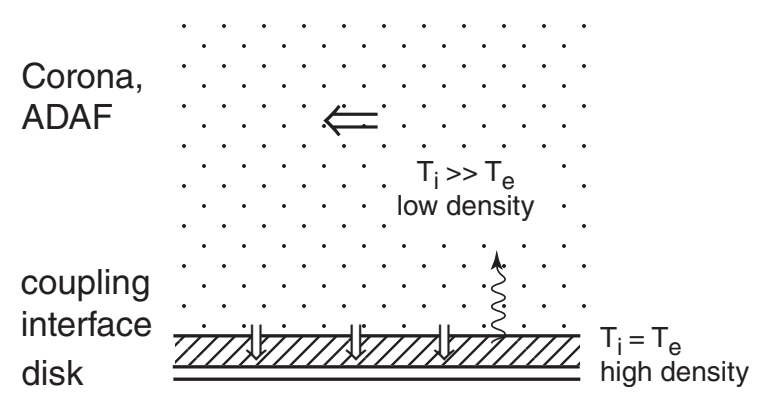

Fig. 2. The hot two-temperature ADAF together with the radiating onetemperature layer below the coupling interface.

This situation changes if there is a disk below the ADAF. Due to the large temperature difference between the hot ADAF and the cool disk, thermal conduction results in further cooling of the electrons. While the ion temperature is not much affected, the electron temperature drops with height until, near the bottom, a temperature $T_{\mathrm{e}}=T_{\mathrm{cpl}}$ is reached at which coupling between ions and electrons becomes efficient, and from then on ion and electron temperatures are the same, $T_{i}=T_{\mathrm{e}}=T$, as illustrated in Fig. 2.

The electron temperature has practically no influence on the dynamics of the flow and the thermodynamics of the ion gas and is subrelativistic, $k T_{\mathrm{e}}<m_{\mathrm{e}} c^{2}$ ( $k$ Boltzmann constant, $m_{\mathrm{e}}$ electron mass, $c$ speed of light). The rate of energy transfer from ions to electrons is given by Stepney (1983). Since in the twotemperature advection-dominated hot flow the ions are at a much higher temperature than the electrons a simplified formula (Liu et al. 2002) can be used

$$
\begin{aligned}
q_{\text {ie }} & =3.59 \times 10^{-32} \mathrm{~g} \mathrm{~cm}^{5} \mathrm{~s}^{-3} \mathrm{deg}^{-1} n_{\mathrm{e}} n_{i} T_{i}\left(\frac{k T_{\mathrm{e}}}{m_{\mathrm{e}} c^{2}}\right)^{-\frac{3}{2}} \\
& =1.05 \times 10^{35} \mathrm{~g} \mathrm{~cm}^{-1} \mathrm{~s}^{-3} \mathrm{deg}^{3 / 2} T_{\mathrm{e}}^{-3 / 2} \alpha^{-2} m^{-2} \dot{m}^{2} r^{-4} .
\end{aligned}
$$

Coupling of ions and electrons is reached when viscous and compressive heating of the ions is balanced by the collisional heat transfer from the ions to the electrons, $q_{\mathrm{ie}}=q^{+}+q^{c}$. This then determines the coupling temperature $T_{\mathrm{cpl}}$. Since the coupling occurs at low height the density there is higher than the height averaged value (Eq. (2)), by a factor $2 / \sqrt{\pi}$ (vertical density distribution $\left.n=n_{0} \exp \left(-z^{2} / H^{2}\right)\right)$. This yields the coupling temperature as

$T_{\text {cpl }}=1.98 \times 10^{9} \alpha^{-4 / 3} \dot{m}^{2 / 3}=1.24 \times 10^{9} \mathrm{~K}$,

the numerical value for $\alpha=0.2$ and $\dot{m}=0.02$ (see Sect. 8.1).

In the case considered here, conductive cooling limits the peak electron temperature in the ADAF to a value $T_{m}$ somewhat lower than the limit where radiation losses balance collisional heating. The synchrotron radiation is optically thick, which makes the synchrotron radiation losses drop steeply with temperature (Narayan \& Yi 1995; Mahadevan 1997). As bremsstrahlung also decreases with temperature we may neglect to a first approximation the radiation losses and calculate the resulting thermal electron profile from the balance between thermal conduction and collisional heating alone,

$$
\begin{aligned}
F_{\mathrm{c}} & =-\kappa_{0} T_{\mathrm{e}}^{5 / 2} \mathrm{~d} T_{\mathrm{e}} / \mathrm{d} z, \\
\frac{\mathrm{d} F_{\mathrm{c}}}{\mathrm{d} z} & =-q_{\mathrm{ie}}\left(T_{\mathrm{e}}\right) .
\end{aligned}
$$

For the thermal conductivity coefficient $\kappa_{0}$ we take the standard value (for the effect of $\kappa_{0}$ on the coronal structure see
Meyer \& Meyer-Hofmeister 2006). Formally multiplying the left sides and the right sides of these two equations by each other and multiplying by $\mathrm{d} z$ reduces this to a first order differential equation for $F_{\mathrm{c}}\left(T_{\mathrm{e}}\right)$ with the solution

$F_{\mathrm{c}}^{2}=\kappa_{0}\left(K n_{i} n_{\mathrm{e}} T_{i}\right)\left(T_{\mathrm{m}}^{2}-T_{\mathrm{e}}^{2}\right)$

with $K=1.64 \times 10^{-17} \mathrm{~g} \mathrm{~cm}^{5} \mathrm{~s}^{-3} \mathrm{deg}^{1 / 2}$. The integration constant $T_{m}$ is the maximal electron temperature reached with height. Note that the downward directed heat flow has a negative value. Integration of $\mathrm{d} z / \mathrm{d} T_{\mathrm{e}}$ (Eq. (6)) using Eq. (8) yields the electron temperature profile in the ADAF,

$z_{\mathrm{m}}-z=\sqrt{\frac{\kappa_{0}}{K n_{i} n_{\mathrm{e}} T_{i}}} T_{\mathrm{m}}^{3 / 2} \int_{T_{\mathrm{e}} / T_{\mathrm{m}}}^{1} \frac{x^{5 / 2}}{\sqrt{1-x^{2}}} \mathrm{~d} x$.

For the low height $z=z_{\mathrm{cpl}}$ where $T_{\mathrm{e}}$ has dropped to the coupling temperature $T_{\mathrm{cpl}}$, in Eq. (9) we may neglect $z_{\mathrm{cpl}}$ compared to $z_{\mathrm{m}}$, and with $T_{\mathrm{cpl}}^{2} / T_{\mathrm{m}}^{2}$ small compared to one, the integral may be extended from zero to one to give 0.719. Equation (9) then yields the maximal electron temperature in the ADAF,

$T_{\mathrm{m}}^{5 / 2}=1.39 z_{\mathrm{m}}\left(K n_{i} n_{\mathrm{e}} T_{i} / \kappa_{0}\right)^{1 / 2}$.

For the height $z_{\mathrm{m}}$ one may take the vertical scale height for $n_{\mathrm{e}} n_{i}$ which is $1 / \sqrt{2}$ of the density scale height $c_{\mathrm{S}} / \Omega_{\mathrm{K}}\left(\Omega_{\mathrm{K}}\right.$ Kepler angular velocity) of Narayan et al. (1998),

$z_{\mathrm{m}}=1.27 \times 10^{5} \mathrm{mr} \mathrm{cm}$.

With this value we obtain $T_{\mathrm{m}}$ from Eq. (10). Equation (8), neglecting $T_{\mathrm{cpl}}^{2}$ compared to $T_{\mathrm{m}}^{2}$, finally yields the thermal flux drained from the ADAF that enters the coupling interface at $z=z_{\mathrm{cpl}}$ from above,

$F_{\mathrm{c}}^{\mathrm{ADAF}}=-\kappa_{0}^{3 / 10}\left(K n_{i} n_{\mathrm{e}} T_{i}\right)^{7 / 10}\left(1.39 z_{\mathrm{m}}\right)^{2 / 5}$

\section{The coupling interface}

In the case of no mass exchange between ADAF and disk, Eq. (12) gives the heat flux that enters at $z=z_{\text {cpl }}$ into the radiating layer from the ADAF above. This is the borderline case considered by Liu et al. (2006). However if mass condenses from the ADAF onto the disk it carries the high thermal heat content of the ADAF ions to the interface. Energy conservation across the interface yields

$F_{\mathrm{cpl}}+\dot{m}_{z} \frac{\gamma}{\gamma-1} \frac{1}{\beta}\left(\frac{\mathfrak{R} T}{\mu}\right)=F_{\mathrm{c}}^{\mathrm{ADAF}}+\dot{m}_{z} \frac{\gamma}{\gamma-1} \frac{1}{\beta}\left(\frac{\mathfrak{R} T_{i}}{\mu_{i}}\right)$.

The factor $\frac{1}{\beta}$ arises when we include magnetic work and internal energy advection together with the corresponding gas terms in the simplified ADAF parameterization of the magnetic field. Here $F_{\mathrm{cpl}}$ is the heat flow leaving the interface on the lower side, $\mathfrak{R}$ is the gas constant, $T$ is the common temperature of ions and electrons on that side, and $\dot{m}_{z}$ is the mass flow rate of the condensing gas per unit area. (It has the dimension of $\mathrm{g} \mathrm{cm}^{-2} \mathrm{~s}^{-1}$ and should not be confused with the non-dimensional ADAF mass accretion rate $\dot{m}$ and similar quantities defined later.) In condensation flow it has a negative value. The heat flow $F_{\text {cpl }}$ entering the radiating layer below the ADAF from above is thus increased over $F_{\mathrm{c}}^{\mathrm{ADAF}}$,

$F_{\mathrm{cpl}}=F_{\mathrm{c}}^{\mathrm{ADAF}}\left[1-\frac{\dot{m}_{z}}{\dot{m}_{z}^{*}}\right]$ 


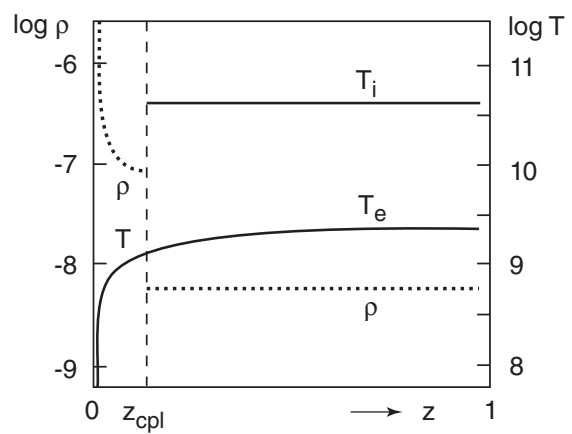

Fig. 3. Changes of ion temperature, electron temperature and density at the distance of 80 Schwarzschild radii and for a mass flow rate of $\dot{m}=0.02$ in the ADAF. The vertical height $z$ is measured in units of the vertical scale height for $n_{\mathrm{e}} n_{i}$.

where

$\dot{m}_{z}^{*}=\frac{\gamma-1}{\gamma} \beta \frac{-F_{\mathrm{c}}^{\mathrm{ADAF}}}{\frac{\mathfrak{R} T_{i}}{\mu_{i}}(1-\epsilon)}$

is a normalization mass flow rate per unit area (positive and of the same physical dimension as $\dot{m}_{z}$ ). It depends on the ADAF parameters. The ratio

$\epsilon=\frac{\mu_{i} T_{\mathrm{cpl}}}{\mu T_{i}}$

is a small number, typically of the order of $10^{-2}$. An example for temperature and density distributions is shown in Fig. 3.

(In the case of evaporation $\left(\dot{m}_{z}>0\right)$ the situation at the boundary between the radiating layer and the ADAF is different. While for a descending flow, collisional coupling immediately heats the electrons and adds to their thermal flux, in an ascending flow, on passing through the boundary, the ions becoming uncoupled must first be heated up by friction to the ADAF temperature before they are fully incorporated in the ADAF. Here we consider only the condensation case.)

\section{The radiating layer below the ADAF}

The parameters of the ADAF determine what happens in the layer between the coupling interface and the disk surface.

Case (1): if the pressure is high, the density in the lower layer also is high. Then the conductive flux drained from the ADAF is already efficiently radiated away at some height before the disk surface is reached. Radiative cooling however continues and must be served by additional heat released from gas descending through the temperature profile to the disk surface, i.e. matter condenses from the ADAF onto the disk.

Case (2): if the pressure is low, only part of the conductive flux can be radiated away; the remaining part is taken up by cool disk matter heating up as it rises through the temperature profile, i.e. matter evaporates from the disk into the corona.

A borderline case in between, case (3), occurs if the ADAF pressure allows the thermal heat flux drained from the ADAF to be radiated away exactly on reaching the disk surface. Then there is neither condensation nor evaporation, i.e. no mass is exchanged between disk and ADAF.

\subsection{The energy balance in the radiating layer}

We derive the condition for the different cases from the energy balance in the radiating layer (compare Liu et al. 2002). We use a simplified form of the energy equation, keeping only the dominant contribution of internal heat, pressure work, and thermal conduction, together with bremsstrahlung cooling $n_{\mathrm{e}} n_{i} \Lambda(T)$,

$\frac{\mathrm{d}}{\mathrm{d} z}\left[\dot{m}_{z} \frac{\gamma}{\gamma-1} \frac{\mathfrak{R} T}{\mu}+F_{\mathrm{c}}\right]=-n_{\mathrm{e}} n_{i} \Lambda(T)$.

We express density by temperature and (constant) gas pressure $\beta p$. This value is taken at the bottom of the ADAF region, and, as for the density, is slightly higher than the vertical mean pressure (Eq. (2)). Assuming free-free radiation for $T_{\mathrm{e}} \geq 10^{7.5} \mathrm{~K}, n_{\mathrm{e}} n_{i} \Lambda(T)$ becomes $\frac{0.25}{k^{2}}(\beta p)^{2} b T^{-3 / 2}$ with $b=$ $10^{-26.56} \mathrm{~g} \mathrm{~cm}^{5} \mathrm{~s}^{-3} \mathrm{deg}^{-1 / 2}$ (Sutherland \& Dopita 1993). For simplicity we use this law also for smaller $T$. The justification is that because of the very steep temperature profile below $10^{7.5} \mathrm{~K}$ such regions contribute only a negligible amount to cooling of this layer (Liu et al. 1995). Contributions of gravitational energy release, frictional heating, and side-wise advection of mass and energy can be neglected for a small extent of this layer. Likewise, kinetic energy is negligible since at high density the flow is highly subsonic.

To solve the second-order differential equation Eq. (17), we use $T$ as the independent variable and define a new dependent variable $g(T) \equiv \kappa_{0} T^{3 / 2} \mathrm{~d} T / \mathrm{d} z=-F_{\mathrm{c}} / T$. We now obtain the firstorder differential equation

$$
\begin{aligned}
g \frac{\mathrm{d} g}{\mathrm{~d} \ln T} & =\frac{0.25 \beta^{2} p^{2}}{k^{2}} \kappa_{0} b+\dot{m}_{z} \frac{\gamma}{\gamma-1} \frac{\mathfrak{R}}{\mu} g-g^{2} \\
& =-\left(g-g_{1}\right)\left(g-g_{2}\right),
\end{aligned}
$$

with

$g_{1}=\frac{\dot{m}_{z}}{2} \frac{\gamma}{\gamma-1} \frac{\mathfrak{R}}{\mu}+\sqrt{\left(\frac{\dot{m}_{z}}{2} \frac{\gamma}{\gamma-1} \frac{\mathfrak{R}}{\mu}\right)^{2}+\frac{0.25 \beta^{2} p^{2}}{k^{2}} \kappa_{0} b}$

and $g_{2}$ differing from $g_{1}$ only by the sign of the square root.

This equation has to be solved with the upper and lower boundary conditions $g=\frac{-F_{\mathrm{cpl}}}{T_{\mathrm{cpl}}}$ at $T=T_{\mathrm{cpl}}$ and $F_{\mathrm{c}}=0$ at $T=0$. In reality, the temperature does not drop to zero at the disk surface but the solution is practically independent of the exact values at the lower boundary (except for a very narrow range in $z$ at the bottom) as long as flux and temperature there become small compared to those at the upper boundary, with no consequence for our results. $\dot{m}_{z}$ is the Eigenvalue to be determined.

The only solution that fulfills the lower boundary condition is the singular solution $g(T)=g_{1}=$ const., i.e. a linear relation between $-F_{\mathrm{c}}$ and $T$. The ratio of these two quantities at the upper boundary determines the value of $g_{1}$. This requires

$\dot{m}_{z}=\frac{\gamma-1}{\gamma} \frac{-F_{\mathrm{c}}^{\mathrm{ADAF}}\left(1-\frac{\dot{m}_{z}}{\dot{m}_{z}^{*}}\right)}{\frac{\mathfrak{R} T_{\mathrm{cpl}}}{\mu}}\left[1-C \frac{1}{1-\left(\frac{\dot{m}_{z}}{\dot{m}_{z}^{*}}\right)^{2}}\right]$

with

$C=\kappa_{0} b\left(\frac{0.25 \beta^{2} p^{2}}{k^{2}}\right)\left(\frac{T_{\mathrm{cpl}}}{F_{\mathrm{c}}^{\mathrm{ADAF}}}\right)^{2}$.

Equations (20), (15), and (16) yield a quadratic equation for $\dot{m}_{z}$,

$\left(1-\frac{\dot{m}_{z}}{\dot{m}_{z}^{*}}\right)^{2}-\epsilon\left(1-\frac{\dot{m}_{z}}{\dot{m}_{z}^{*}}\right)-(1-\epsilon) C=0$,

with the solution

$\frac{\dot{m}_{z}}{\dot{m}_{z}^{*}}=1-\frac{\epsilon}{2}-\sqrt{\frac{\epsilon 2}{4}+(1-\epsilon) C}$. 
The quantity $C$ of Eq. (21) compares the radiation loss in the thermal profile with the heat flux drained from the ADAF. For $C=1$ Eq. (20) gives $m_{z}=0$, the borderline case discussed by Liu et al. (2006). For $C<1$, the radiation losses are too weak, $\dot{m}_{z}$ is positive and the heat flow is used up to heat the evaporating gas to the (un-)coupling boundary temperature $T_{\mathrm{cpl}}$. If, on the other hand, $C>1$, the gas in this layer is efficiently cooled, sinks down and condenses.

\section{The mass exchange between ADAF and disk}

\subsection{The borderline case: no mass exchange, $C=1$}

The value of $C$ depends on the ADAF parameters $\alpha, \beta, \dot{m}$ and $r$, but not on $m$. This shows that the same situation holds for supermassive and stellar black holes alike. Also note a significant dependence on $\beta, C$ scales almost with $\beta^{2}$ (Eq. (21)). For $\beta=0.8$ we get

$C=0.96 \alpha^{-28 / 15} \dot{m}^{8 / 15} r^{-1 / 5}$.

The radius $r=r_{\mathrm{c}}$ at which $C=1$ marks the transition between outside evaporation and inside condensation of an inner disk below the ADAF,

$r_{\mathrm{c}}=0.815 \alpha^{-28 / 3} \dot{m}^{8 / 3}$.

Here, we take $\alpha=0.2$ (corresponding to $\alpha=0.3$ in ShakuraSunyaev notation) but note the extremely strong dependence of $r_{\mathrm{c}}$ on the choice of this parameter. Since $\alpha$ incorporates several underlying physical processes of unresolved detail its exact value is not known. Various values of the above order of magnitude are quoted in the literature (see Narayan et al.1998). The re-condensation process discussed here occurs in connection with spectral state transitions for which Maccarone (2003) found $\dot{m}=0.02$ from a detailed investigation of these transitions in X-ray binaries. For these values $C$ becomes 1 at a distance of about 80 Schwarzschild radii, an order of magnitude discussed for inner disks in intermediate states (Ibragimov et al. 2005). The dependence of $C$ on mass flow rate and distance obtained allows us to derive conditions for the existence of an inner disk below the ADAF.

\subsection{Condensation in the inner region}

It is an interesting question as to how the fraction of the ADAF that re-condenses onto the inner disk and then flows inward via the disk depends on the ADAF parameters. For this we add all contributions from the distance $R_{\mathrm{c}}=r_{\mathrm{c}} R_{\mathrm{S}}$ where condensation begins down to an inner cut-off radius $R_{\mathrm{i}}=r_{\mathrm{i}} R_{\mathrm{S}}$, e.g. the radius of the innermost stable orbit around a non-rotating black hole, $r_{\mathrm{i}}=3$. As discussed later, the inner edge may well lie at some larger distance. Like the ADAF accretion rate, the total condensation rate will be measured in Eddington accretion rate. (In our definition condensation rates have a negative sign.) Using Eq. (20), (for $\epsilon \ll 1$ ) the total rate of condensation onto both sides of the disk becomes

$$
\begin{aligned}
\left|\dot{m}_{\text {cond }}\right| & =\int_{R_{\mathrm{i}}}^{R_{\mathrm{c}}} \frac{4 \pi R^{2}}{\dot{M}_{\mathrm{Edd}}} \dot{m}_{z} \frac{\mathrm{d} R}{R} \\
& =\int_{r_{\mathrm{i}}}^{r_{\mathrm{c}}} \dot{m}_{\mathrm{D}}^{*}(\sqrt{C}-1) \frac{\mathrm{d} r}{r}
\end{aligned}
$$

with

$$
\dot{m}_{\mathrm{D}}^{*}=\frac{4 \pi R^{2} \dot{m}_{z}^{*}}{\dot{M}_{\mathrm{Edd}}}=8.31 \times 10^{-3} \alpha^{-7 / 5} \dot{m}^{7 / 5} r^{3 / 5} \text {. }
$$

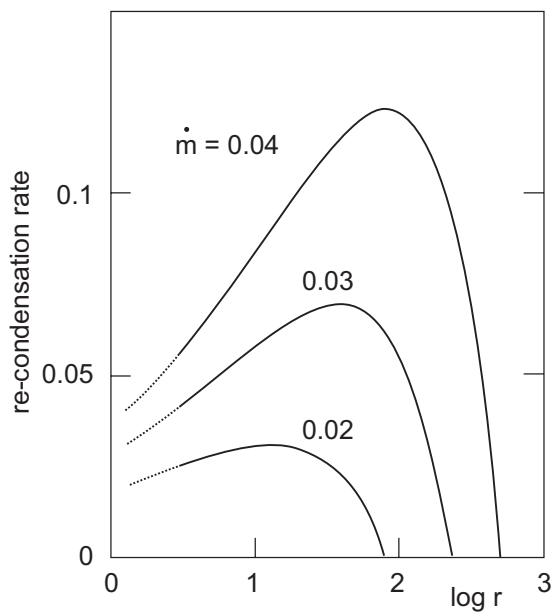

Fig. 4. Re-condensation of gas from the ADAF onto an inner disk as function of the distance $r$ (in units of $R_{\mathrm{S}}$ ) for various mass flow rates $\dot{m}$ in the ADAF (in units of $\dot{M}_{\text {Edd }}$ ). The curves display the spatial distribution of contributions of logarithmic intervals in $r$ as areas below the curve. The ordinate is re-condensation rate relative to the mass flow rate in the ADAF. Re-condensation occurs at a larger distances for higher $\dot{m}$. Below $3 R_{\mathrm{S}}$ the curves are dotted.

The integrand $\dot{m}_{\mathrm{D}}^{*}(\sqrt{C}-1)$ gives the contribution of logarithmic intervals in r. Its distribution is shown in Fig. 4 for various values of $\dot{m}$. The ordinate is the integrand relative to the ADAF mass flow rate $\dot{m}$. The area underneath each graph gives the relevant total condensation rate.

Introducing $x=r / r_{\mathrm{c}}$ instead of $r$ in Eq. (26) and making use of Eqs. (24), (25), and (28) one can evaluate the integral and obtain the total re-condensation rate as

$\frac{\left|\dot{m}_{\text {cond }}\right|}{\dot{m}}=2.34 \times 10^{-2} \alpha^{-7} \dot{m}^{2} f\left(R_{\mathrm{i}} / R_{\mathrm{c}}\right)$

with

$f(x)=1-6 x^{1 / 2}+5 x^{3 / 5}$.

The function $f$ approaches 1 when re-condensation begins at a radius $R_{\mathrm{c}}$ that is large compared to the inner cut-off radius $R_{\mathrm{i}}$. As the ADAF mass flow rate decreases $R_{\mathrm{c}}$ moves inward, $f$ and the re-condensation rate, i.e. the mass flow rate in the inner disk decrease until at a certain lower ADAF mass flow rate the outer disk edge $R_{\mathrm{c}}$ reaches $R_{\mathrm{i}}$ where $f$ becomes 0 and the disk size has shrunk to zero. For even lower mass flow rates there is no recondensation and the inner disk disappears. This intriguing behavior appears able to explain why Cyg X-1 does not show the hysteresis between hard/soft and soft/hard spectral state transitions that all transient X-ray sources display. We discuss this in the next section.

\subsection{Complete re-condensation?}

The model results depend on the parameters used to describe the ADAF. The dependence on the parameters $\alpha$ and $\dot{m}$ is particularly strong for the re-condensation rate (Eq. (29)) and the radius at which re-condensation begins, i.e. the outer radius of an inner disk below the ADAF (Eq. (25)). In the example given above both values are rather small. However, a small decrease of $\alpha$ and a slight increase of $\dot{m}$ can bring these values to $100 \%$ re-condensation with re-condensation beginning already at 1000 Schwarzschild radii (this is an extrapolation as our integral Eq. (26) neglects changes of $\dot{m}$ by re-condensation). 
This is relevant for standard disk evaporation models (e.g. Meyer et al. 2000). They predict that at distances of a few hundred to a thousand Schwarzschild radii there is a significant mass flow rate in an accretion disk corona, of a few percent of the Eddington value. If the mass accretion rate drops below that value, all the mass evaporates into the corona and from there on flows inward as a pure coronal flow, i.e. an ADAF. This yields a standard model for spectral state transitions. If the mass flow rate in the disk exceeds that critical value the disk is not truncated and continues to the last stable orbit. This accretion mode gives the characteristic soft state spectrum of a multi-temperature black body. But even in this case the rather strong coronal flow at the distances of a few hundred Schwarzschild radii must exist. Why does it not flow on as a 2-temperature ADAF above the disks and shows as a significant hard contribution to the soft state spectra? The answer from our intermediate state analysis is that recondensation becomes complete for mass accretion rates as high as the coronal evaporation rate at a few hundred Schwarzschild radii. This explanation connects the processes of disk truncation and re-condensation. The upper part of Fig. 1 illustrates the situation.

\subsection{Hysteresis}

Even for lower mass accretion rates, when the outer disk already is truncated, re-condensation onto the remaining inner disk may be close to complete and still yield an apparent soft state in the innermost region. This extends the soft state to a lower mass accretion rate on the declining part of an outburst and leads to lower luminosity for the transition from the soft to the hard spectrum in decline than for the opposite transition during the rise, i.e. hysteresis. Such hysteresis would be independent of, and add to the irradiation caused hysteresis referred to in Sect. 9.

\section{Cyg X-1, a black hole accretor without hysteresis}

Cyg X-1 shows only moderate changes in the mass accretion rate, attributed to the fact that it is a wind accretor. This is very different from transient X-ray sources where the accretion rate increases and decreases 100 fold during an outburst. Cyg X-1 stays in the hard state most of the time, but several spectral transitions to the soft state were observed. Because of the narrow range in mass accretion rate the hard state of Cyg X-1 is still intermediate in nature as the observations of Ibragimov et al. (2005) show.

Also by another fact Cyg X-1 differs from the transient sources. There is no noticeable hysteresis in Cyg X-1 (Zdziarski et al. 2002; Zdziarski \& Gierliński 2005). This was pointed out as a problem also by Lachowicz \& Czerny (2005). Lightcurves of transient sources show a clear hysteresis in the transition luminosities (the soft/hard transition occurs at luminosities lower by a factor of 3 to 5 than the hard/soft transition, e.g. in Aql X-1 (Maccarone \& Coppi 2003), GX 339-4 (Miyamoto et al. 1995; Nowak et al. 2002; Zdziarski et al. 2004), XTE J1650-500 (Rossi et al. 2003), XTE J1500-564 (Rodrigues et al. 2003). The different luminosities at spectral transition can be understood as due to different maximal evaporation rates in the two states (triggering the transition) for either hard or soft irradiation of the corona by the central source (Meyer-Hofmeister et al. 2005).

The new feature discussed in our work here, an inner recondensation disk, offers a solution for the mystery of why Cyg X-1 has no hysteresis while all the transients have it.

In transient sources the re-condensation disk recedes inward and finally disappears completely as the mass accretion rate continues to fall, as is the case in the decline from outburst into quiescence. Then, no inner disk remains on which recondensation could occur when the system moves into the next outburst. The spectrum remains hard at increasing luminosity. Only when the accretion rate reaches the higher critical value for which the transition under hard irradiation occurs does it become soft.

Cyg $\mathrm{X}$ is different. When that system changes from a soft state excursion back to its usual hard state it does so with only a modest decrease of the mass accretion rate and never falls into deep quiescence. This allows an inner re-condensation disk to survive as the observations indicate. When now another episode of transition to a soft state occurs the mass accretion rate rises, re-condensation increases, the inner disk expands and carries an increasing disk mass flow until it smoothly merges with the outer disk to form the standard soft accretion state. Together with recondensation the irradiation is completely reversible and no hysteresis occurs. This explanation relates the special character of the wind accretor to the otherwise enigmatic feature of "no hysteresis" in a natural way and seems a strong support for the re-condensation model.

\section{Discussion}

A more detailed numerical investigation of the re-condensation process is desirable. The present simple analytical model might serve to show the features and their dependence on the parameters to be expected in this interesting accretion mode.

\subsection{Interpretation of the intermediate state}

When the mass accretion rate gradually decreases further as a source declines from outburst the re-condensation rate onto the receding inner disk declines, a significant part of the accretion flow stays in the ADAF and shows characteristics of the hard state but modified by the existence of cool material in the form of a weakly accreting inner disk. From our analysis we find that the intermediate states during the outburst decline last longer for a slow decrease of the mass flow rate and less time for a fast decrease.

Observations for transient black hole X-ray sources show those features. During the slow luminosity decline after the 1999 outburst of XTE J1859+226 (Corbel et al. 2004, Fig. 9) the spectrum was classified as due to an intermediate state. The steep luminosity decline of GX 339-4 in 2003 (Remillard 2005, Fig. 3) is related to the very short intermediate state.

Short intermediate states of a few days might be caused by an inner disk disappearing in its viscous time. Similar to these short states is the situation during rise to outburst, where the inner edge of the disk moves inward within a viscous time, only an ADAF inside. If the mass flow rate in the disk is always well below the maximal evaporation rate the disk is truncated all the time, and no thermal conduction affects the ADAF. XTE J1118+480 is a well studied source which stays in the hard state even during outburst. We would expect a disk truncation at several hundred $R_{\mathrm{S}}$. But Esin et al. (2001) from multiwavelength observations concluded that the disk truncation lies at about $55 R_{\mathrm{S}}$ in outburst. How can this be understood? The highest mass flow rate during outburst was found to be about $0.02 \dot{M}_{\mathrm{Edd}}$, the value generally accepted for state transition (Maccarone 2003). This means that the mass flow rate could have surpassed the critical rate for a short while and the outer disk began to extend inward. Re-condensation immediately would begin. This re-condensation can then continue even as the accretion rate drops below the critical rate, leading to an intermediate state. 


\subsection{Inner edge of the disk?}

The disk truncation in general varies with the mass accretion rate. For low luminosity sources, X-ray binaries or AGN in a hard spectral state, the inner edge of the outer disk was determined by fitting multiwavelength spectra, understood as caused by an ADAF and an outer disk. For very low accretion rates large truncation radii were found, of the order of $10^{3}$ to $10^{4} R_{\mathrm{S}}$ (e.g. Esin et al. 1997; Narayan et al. 1997; Narayan 2005). For X-ray binaries with higher mass accretion rates in phases of rise to an outburst or decay, from a multicolor disk model smaller values for the inner disk radius were deduced (compare Sobczak et al. 2000; see examples in the review of McClintock $\&$ Remillard 2006). In a recent detailed investigation of the spectrum of Cyg X-1 Ibragimov et al. (2005) found truncation radii around $50-100 R_{\mathrm{S}}$. Note that the mass flow rate was not much below the transition rate, i.e. a typical intermediate state.

Comparing with the predictions of the standard evaporation model we find good agreement for the low luminosity sources. The model also is consistent with no disk truncation for high mass flow rates.

For the intermediate state of Cyg X-1, Ibragimov et al. (2005) analyzed spectral observations in terms of Compton reflection, the index of the hard power law spectrum, the width of the $\mathrm{Fe} \mathrm{K} \alpha$ line, and an additional soft excess of a few $\mathrm{keV}$ of unknown origin. With the correlation between photon index and amplitude of reflection they inferred disk cut-off distances of 50-100 $R_{\mathrm{S}}$, varying with the accretion rate. Esin et al. (2001) inferred a similar truncation at $55 R_{\mathrm{S}}$ for XTE J1118+480 in an apparently similar hard (-intermediate) state (see the discussion in the preceding subsection).

On the other hand, Miller et al. (2006a) argue for an untruncated disk extending to the last stable orbit during the lowhard state of the 2004 outburst of GX 339-4, at a luminosity of $5 \%$ of the Eddington value. From a preliminary analysis of data from SWIFT J17335-0127 in the low-hard state of its 2005 outburst, Miller et al. (2006b) see indication for a similarly untruncated disk, here at a luminosity of one third of a per cent of the Eddington value. (Note that in optical thin emission the accretion luminosity can be lower than the corresponding accretion rate, both measured in their Eddington units.) This would suggest an untruncated re-condensation disk in these systems. The situation deserves further clarification.

The re-condensation model discussed here leaves open the question of if and where a remaining inner disk may itself be truncated. Neglected terms in the analysis, different assumptions about $\gamma$, and a more detailed calculation all can affect the outcome of the re-condensation rate estimates. One also cannot exclude that a parameter used to describe the ADAF varies with distance. The ratio of magnetic pressure to gas pressure, for example, could increase at smaller radii as accretion carries magnetic flux inwards. This decreases $\beta$ and $C$ (Eq. (21)). If $C$ falls below one (Eq. (23)), condensation turns into evaporation and the disk can become truncated before the final last stable orbit is reached. The effect of disk magnetic fields on evaporation in the standard model is discussed in Meyer \& Meyer-Hofmeister (2002).

\subsection{Intermediate spectral states in disks around supermassive black holes?}

The solutions discussed in this paper are independent of the mass $m$ of the black hole. Thus they should be applicable also to accretion on supermassive black holes in AGN. Recently
Jester (2005) studied the distribution of AGN bolometric luminosities and black hole masses for objects from the SDSS spectroscopic quasar survey to test the existence of two different accretion modes (as predicted theoretically, Narayan et al. 1998) and found a change of mode at an Eddington-scaled accretion rate of about 0.01 . Markowitz \& Uttley (2005), comparing power density functions pointed out the analogy of low luminosity AGN to low/hard state black hole X-ray binaries. This suggests that some of the AGN accretors should be found in an intermediate state like their galactic stellar mass counterparts. To actually observe a transition through the different accretion states might be difficult as dynamical and viscous timescales scale with the mass of the black hole. Yuan et al. (2004) compared ROSAT and XMM Newton observations of 386 sources and found in one of them, the Seyfert-LINER galaxy NGC 7589, an increase in X-ray flux by a factor of $>10$ over a time of 5 years, a timescale of the order of the diffusion time at a distance of a few hundred Schwarzschild radii from a $10^{7} M_{\odot}$ black hole. As the XMM high state luminosity was estimated to be a few percent of the Eddington value the authors suggest that the system might have been found during a spectral transition.

\section{Conclusions}

We have presented a simple analytical model for re-condensation of gas from an ADAF onto a disk below under the action of thermal conduction and radiation. The model is able to explain how cool gas can exist in the close neighborhood of an accreting black hole, after a standard accretion disk has become truncated and an ADAF has formed, a situation that occurs when the mass flow rate decreases during the transition from soft to hard spectral state of galactic black hole X-ray binaries. A fairly complete re-condensation of the ADAF into the disk would extend an inner thin disk accretion with its soft spectral state to lower mass accretion rates, and suggests a hysteresis effect independent of and in addition to the irradiation effect discussed earlier. The model also clarifies why in soft state the considerable coronal flow at distances of several hundred Schwarzschild radii predicted theoretically does not continue as a hot 2-temperature flow to the interior. Such a flow would appear as a hard contribution in the spectrum that is not observed. The model receives considerable support from being able to resolve the mystery of why Cyg X-1 does not show hysteresis between the luminosity at soft/hard and hard/soft spectral transition that all X-ray transient sources display.

This simple model deserves further, more detailed investigation.

Acknowledgements. We thank Marat Gilfanov for helpful discussions. B. F. Liu acknowledges support by the National Natural Science Foundation of China (NSF-10533050) and the BaiRenJiHua program of the Chinese Academy of Sciences.

\section{References}

Barret, D., \& Olive, J.-F. 2002, ApJ, 576, 391

Belloni, T., Homan, J., Casella, P., et al. 2005, A\&A, 440, 207

Chen, W., Shrader, C. R., \& Livio, M. 1997, ApJ, 491, 312

Corbel, S., Fender, R. P., Tomsick, J. A., et al. 2004, ApJ, 617, 1272

Cui, W., Zhang, S. N., Focke, W., et al. 1997, ApJ, 484, 383

Duschl, W. J. 1986, A\&A, 163, 61

Esin, A. A. 1997, ApJ, 482, 400

Esin, A. A., McClintock, J. E., \& Narayan, R. 1997, ApJ, 489, 865

Esin, A. A., McClintock, J. E., Drake, J. J., et al. 2001, ApJ, 555, 483

Fender, R. P., Belloni, T. M., \& Gallo, E. 2004, MNRAS, 355, 1105

Gallo, E., \& Fender, R. P. 2005, Mem. S. A. It., 76, 600 
Gierliński, M., \& Newton, J. 2006, MNRAS, 370, 837

Göğüs, E., Finger, M. H., Kouveliotou, C., et al. 2004, ApJ, 609, 977 Homan, J., Klein-Wolt, M., Rossi, S., et al. 2003, ApJ, 586, 1262 Ibragimov, A., Poutanen, J., Gilfanov, M., et al. 2005, MNRAS, 362, 879 Jester, S. 2005, ApJ, 625, 667

Kalemci, E., Tomsick, J. A., Rothschild, R. E., et al. 2003, ApJ, 586, 419 Lachowicz, P., \& Czerny, B. 2005, MNRAS, 361, 645

Liu, B. F., Meyer, F., \& Meyer-Hofmeister, E. 2005, A\&A, 442, 555

Liu, B. F., Meyer, F., \& Meyer-Hofmeister, E. 2006, A\&A, 454, L9

Liu, F. K., Meyer, F., \& Meyer-Hofmeister, E. 1995, A\&A, 300, 823

Liu, B. F., Mineshige, S., Meyer, F., et al. 2002, ApJ, 575, 117

Maccarone, T. J. 2003, A\&A, 409, 697

Maccarone, T. J., \& Coppi, P. S. 2003, MNRAS, 338, 189

Mahadevan, R. 1997, ApJ, 477, 585

Markowitz, A., \& Uttley, P. 2005, ApJ, 625, 39

McClintock, J. E., \& Remillard, R. A. 2006, in Compact Stellar X-ray Sources, ed. W. H. G. Lewin, \& M. van der Klis (Cambridge Univ. Press), 157

Meyer, F. 2004, Prog. Theor. Phys. Suppl., 155, 45

Meyer, F., \& Meyer-Hofmeister, E. 1984, A\&A, 132, 143

Meyer, F., \& Meyer-Hofmeister, E. 1994, A\&A, 288, 175

Meyer, F., \& Meyer-Hofmeister, E. 2002, A\&A, 392, L5

Meyer, F., \& Meyer-Hofmeister, E. 2006, A\&A, 449, 443

Meyer, F., Liu, B. F., \& Meyer-Hofmeister, E. 2000, A\&A, 361, 175

Meyer-Hofmeister, E., Liu, B. F., \& Meyer, F. 2005, A\&A, 432, 181

Miller, J. M., Homan, J., Steeghs, D., et al. 2006a, ApJ, 653, 525

Miller, J. M., Homan, J., \& Miniutti, G. 2006b, ApJ, 652, L113

Miyamoto, S., Kitamoto, S., Hayashida, K., et al. 1995, ApJ, 442, L13

Narayan, R. 2005, Astrophys. Space Sci., 300, 177

Narayan, R., \& Yi, I. 1994, ApJ, 428, L13

Narayan, R., \& Yi, I. 1995, ApJ, 452, 710

Narayan, R., Barret, D., \& McClintock, J. E. 1997, ApJ, 482, 448
Narayan, R., Mahadevan, R., \& Quataert, E. 1998, in The Theory of Black Hole Accretion Discs, ed. M. A. Abramowicz et al. (Cambridge Univ. Press), 48 Nowak, M. A., Wilms, J., \& Dove, J. B. 2002, MNRAS, 332, 865

Pottschmidt, K., Chernyakova, M., Zdziarski, A. A., et al. 2006, A\&A, 452, 285 Psaltis, D. 2006, in Compact Stellar X-ray Sources, ed. W. H. G. Lewin, \& M. van der Klis (Cambridge Univ. Press), 1

Remillard, R. A. 2005, in Interacting Binaries: Accretion, Evolution and Outcomes, ed. L. A. Antonelli et al., AIP Conf. Proc., 797, 231

Revnivtsev, M. G., Trudolyubov, S. P., \& Borozdin, K. N. 2000, MNRAS, 312, 151

Rodriguez, J., Corbel, S., \& Tomsick, J. A. 2003, ApJ, 595, 1032

Różańska, A., \& Czerny, B. 2000a, MNRAS, 316, 473

Różańska, A., \& Czerny, B. 2000b, A\&A, 360, 1170

Rossi, S., Homan, J., Miller, J., et al. 2004, in Proc. of the 2nd BeppoSAX Conference, ed. E. P. J. van den Heuvel, J. J. M. in 't Zand, \& R. A. M. J. Wijers, Nucl. Phys. B, Proc. Suppl., 416

Shakura, N. I., \& Sunyaev, R. A. 1973, A\&A, 24, 337

Sharma, P., Hammet, W., Quataert, E., et al. 2006, ApJ, 637, 952

Smith, D. M., Heindl, W. A., Markwardt, C. B., et al. 2001, ApJ, 554, L41

Smith, D. M., Heindl, W. A., \& Swank, J. H. 2002, ApJ, 569, 362

Sobczak, G. J., McClintock, J. E., Remillard, R. A., et al. 2000, ApJ, 544, 993

Stepney, S. 1983, MNRAS, 202, 467

Sutherland, R. S., \& Dopita, M. A. 1993, ApJ, Suppl., 88, 253

Tomsick, J. A., Corbel, S., Goldwurm, A., et al. 2005, ApJ, 630, 413

Yuan, F. \& Zdziarski, A. A. 2005, MNRAS, 354, 953

Yuan, W., Komossa, St., \& Xu, D. 2004, MNRAS, 353, 29

Zdziarski, A. A., \& Gierliński, M. 2004, Prog. Theor. Phys. Suppl., 155, 99

Zdziarski, A. A., Poutanen, J., Paciesasa, W. S., et al. 2002, ApJ, 578, 357

Zdziarski, A. A., Gierliński, M., Mikolajewska, J., et al. 2004, MNRAS, 351, 791 